\title{
Coupled point results in partially ordered metric spaces without compatibility
}

\author{
Fawzia Shaddad ${ }^{1 *}$, Mohd Salmi Md Noorani', Saud M Alsulami² and Habibulla Akhadkulov ${ }^{1}$
}

"Correspondence:
fzsh99@gmail.com
${ }^{1}$ School of Mathematical Sciences,
Faculty of Science and Technology,
Universiti Kebangsaan Malaysia,
UKM, Bangi, Selangor Darul Ehsan
43600, Malaysia
Full list of author information is
available at the end of the article

available at the end of the article

\begin{abstract}
The existence of fixed points, coupled fixed points, and coupled coincidence points without the assumption of compatibility is established. The results presented in this paper extend, improve, and generalize some well-known results in the literature. Also, an example is given to show that our results are real generalizations of known ones in coupled coincidence fixed point theory.
\end{abstract}

MSC: $54 \mathrm{H} 25 ; 47 \mathrm{H} 10$

Keywords: fixed point; coupled fixed point; coupled coincidence point; partially ordered metric space

\section{Introduction}

Fixed point theorems in metric spaces play a major role for solving problems in applied mathematics and science. The study of fixed points of self maps which satisfy certain contractive conditions have been researched extensively by many mathematicians in different directions and in different spaces, we refer the reader to [1-8], and the references therein.

The Banach contraction principle is an important tool in the theory of metric spaces, it guarantees the existence and uniqueness of fixed points of certain self maps of metric spaces.

Recently, Ran and Reurings [9] extended the Banach contraction principle in partially ordered sets with some applications to matrix equations. In [10] Bhaskar and Lakshmikantham introduced the concept of coupled fixed point of a function $F: X \times X \rightarrow X$ which has the mixed monotone property, where $X$ is a partially ordered metric space. Furthermore, they established some interesting coupled fixed point theorems.

After that, Lakshmikantham and Ćirić [11], Choudhury and Kundu [12], Alsulami [13] and many other authors have continued to study the existence of coupled fixed points in partially ordered metric spaces.

Our main aim in this manuscript is to obtain more general fixed point results. This manuscript is split to three stages. First, the existence and uniqueness of fixed points for a complete partially ordered metric spaces have been proved. This result extends the main results of Harjani and Sadarangani [14], Ran and Reurings [9] and Nieto and RodríguezLópez [15]. Second, we establish the existence of coupled fixed point theorems. These results extend and generalize the results of Harjani et al. [16], Bhaskar and Lakshmikantham [10] and Luong and Thuan [17]. In the last section, we give coupled coincidence point theo-

\section{黛 Springer}

( 2014 Shaddad et al.; licensee Springer. This is an Open Access article distributed under the terms of the Creative Commons Attribution License (http://creativecommons.org/licenses/by/2.0), which permits unrestricted use, distribution, and reproduction in any medium, provided the original work is properly cited. 
rems without using the compatibility. Some results of Alsulami [13], Alotaibi and Alsulami [18], Razani and Parvaneh [19] and Lakshmikantham and Ćirić [11] are extended and generalized in the last section. At the end, we illustrate our results by an example where the previous mentioned results cannot be applied.

\section{Fixed point results}

In this section we prove some fixed point results for a mapping $f: X \rightarrow X$ in a partial ordered set $X$ with a metric defined on it.

Let us start with the definition of altering distance.

Definition 2.1 An altering distance function is a function $\psi:[0, \infty) \rightarrow[0, \infty)$ which satisfied the following conditions:

(1) $\psi$ is continuous and nondecreasing;

(2) $\psi(t)=0$ if and only if $t=0$.

Here, the notion of comparability is defined in the following way.

Definition 2.2 Let $(X, \preceq)$ be a partially ordered set. We say that $x, y \in X$ are comparable if $x \preceq y$ or $y \preceq x$ and we write $x \asymp y$.

In [14], Harjani and Sadarangani proved the following interesting results.

Theorem 2.3 Let $(X, d, \preceq)$ be a complete partially ordered metric space and let $f: X \rightarrow X$ be a continuous and nondecreasing mapping such that

$$
\psi(d(f(x), f(y))) \leq \psi(d(x, y))-\varphi(d(x, y)) \quad \text { for } x \succeq y,
$$

where $\psi$ and $\varphi$ are altering distance functions. If there exists $x_{0} \in X$ with $x_{0} \preceq f\left(x_{0}\right)$ then $f$ has a fixed point. Moreover, if for each $x, y \in X$ there exists $z \in X$ such that $x \preceq z$ and $y \preceq z$ then the fixed point is unique.

They also showed that the above theorem is still valid for $f$ not necessarily continuous.

Theorem 2.4 Let $(X, d, \preceq)$ be a complete partially ordered metric space. Assume that if $\left\{x_{n}\right\}$ is a nondecreasing sequence in $X$ such that $x_{n} \rightarrow x$ then $x_{n} \preceq x$ for all $n \in \mathbb{N}$. Let $f:$ $X \rightarrow X$ be a nondecreasing mapping such that

$$
\psi(d(f(x), f(y))) \leq \psi(d(x, y))-\varphi(d(x, y)) \quad \text { for } x \succeq y
$$

where $\psi$ and $\varphi$ are altering distance functions. If there exists $x_{0} \in X$ with $x_{0} \preceq f\left(x_{0}\right)$ then $f$ has a fixed point. Moreover, if for each $x, y \in X$ there exists $z \in X$ such that $x \preceq z$ and $y \preceq z$ then the fixed point is unique.

The following our result is essential for the remaining results. Moreover, this theorem is a generalization of Theorem 2.3 and Theorem 2.4.

Theorem 2.5 Let $(X, d, \preceq)$ be a complete partially ordered metric space. Let $f: X \rightarrow X$ be a mapping which obeys the following conditions: 
(1) there exist an altering distance function $\psi$, an upper semi-continuous function $\theta:[0, \infty) \rightarrow[0, \infty)$, and a lower semi-continuous function $\varphi:[0, \infty) \rightarrow[0, \infty)$ such that

$$
\psi(d(f(x), f(y))) \leq \theta(d(x, y))-\varphi(d(x, y)) \quad \text { for } x \succeq y
$$

where $\theta(0)=\varphi(0)=0$ and $\psi(t)-\theta(t)+\varphi(t)>0$ for all $t>0$;

(2) there exists $x_{0} \in X$ such that $x_{0} \asymp f\left(x_{0}\right)$;

(3) $f$ is nondecreasing;

(4) (a) $f$ is continuous or

(b) if $x_{n} \rightarrow x$ when $n \rightarrow \infty$ in $X$, then $x_{n} \asymp x$ for all $n$.

Then $f$ has a fixed point. Moreover, iffor each $x, y \in X$ there exists $z \in X$ which is comparable to $x$ and $y$ then the fixed point is unique.

Proof By condition (2), there exists a point $x_{0}$ such that $x_{0} \asymp f\left(x_{0}\right)$. Take $x_{1} \in X$ such that $x_{1}=f\left(x_{0}\right)$. That is, $x_{0} \asymp x_{1}$. Take $x_{2}=f\left(x_{1}\right)$. From condition (3), we deduce that $f\left(x_{0}\right) \asymp f\left(x_{1}\right)$, that is, $x_{1} \asymp x_{2}$ again use condition (3) we have $f\left(x_{1}\right) \asymp f\left(x_{2}\right)$. Proceeding by induction, we obtain $x_{n+1}=f\left(x_{n}\right)$ such that $x_{n} \asymp x_{n+1}$ for each $n \in \mathbb{N}$, i.e.,

$$
x_{0} \asymp x_{1} \asymp x_{2} \asymp \cdots \asymp x_{n} \asymp \cdots .
$$

That is,

$$
x_{0} \preceq x_{1} \preceq x_{2} \preceq \cdots \preceq x_{n} \preceq \cdots \quad \text { or } \quad x_{0} \succeq x_{1} \succeq x_{2} \succeq \cdots \succeq x_{n} \succeq \cdots
$$

If $x_{n}=x_{n+1}$ for some $n \in \mathbb{N}$ then $f$ has a fixed point and the proof of the existence of the fixed point is complete. Assume that $x_{n} \neq x_{n+1}$ for all $n \in \mathbb{N}$. Now, by using condition (1), we get

$$
\begin{aligned}
\psi\left(d\left(x_{n}, x_{n+1}\right)\right) & =\psi\left(d\left(f\left(x_{n-1}\right), f\left(x_{n}\right)\right)\right) \\
& \leq \theta\left(d\left(x_{n-1}, x_{n}\right)\right)-\varphi\left(d\left(x_{n-1}, x_{n}\right)\right)
\end{aligned}
$$

but we have $\psi\left(d\left(x_{n-1}, x_{n}\right)\right)-\theta\left(d\left(x_{n-1}, x_{n}\right)\right)+\varphi\left(d\left(x_{n-1}, x_{n}\right)\right)>0$. Then

$$
\frac{\psi\left(d\left(x_{n}, x_{n+1}\right)\right)}{\psi\left(d\left(x_{n-1}, x_{n}\right)\right)} \leq \frac{\theta\left(d\left(x_{n-1}, x_{n}\right)\right)-\varphi\left(d\left(x_{n-1}, x_{n}\right)\right)}{\psi\left(d\left(x_{n-1}, x_{n}\right)\right)}<1 .
$$

Thus

$$
\psi\left(d\left(x_{n}, x_{n+1}\right)\right)<\psi\left(d\left(x_{n-1}, x_{n}\right)\right) .
$$

Since $\psi$ is an altering distance,

$$
d\left(x_{n}, x_{n+1}\right)<d\left(x_{n-1}, x_{n}\right) .
$$

That is, $\left\{d\left(x_{n-1}, x_{n}\right)\right\}$ is decreasing and so

$$
\lim _{n \rightarrow \infty} d\left(x_{n-1}, x_{n}\right)=r .
$$


To show that $r=0$, we suppose that $r \neq 0$. By using the property of $\psi, \theta$, and $\varphi$ and taking $n \rightarrow \infty$ in (2.2) we obtain

$$
\psi(r) \leq \theta(r)-\varphi(r)
$$

So

$$
\psi(r)-\theta(r)+\varphi(r) \leq 0
$$

a contradiction. Therefore, $r=0$ and $\lim _{n \rightarrow \infty} d\left(x_{n-1}, x_{n}\right)=0$.

Now, we want to show that $\left\{x_{n}\right\}$ is a Cauchy sequence. Suppose $\left\{x_{n}\right\}$ is not Cauchy. Then there exists $\varepsilon>0$ and we can find subsequences $\left\{x_{n_{(i)}}\right\}$ and $\left\{x_{m_{(i)}}\right\}$ of $\left\{x_{n}\right\}$ with $n_{(i)}>m_{(i)}>i$, such that

$$
d\left(x_{m_{(i)}}, x_{n_{(i)}}\right) \geq \varepsilon
$$

Suppose that $n_{(i)}$ is the smallest index with $n_{(i)}>m_{(i)}>i$ and satisfying (2.5). This means

$$
d\left(x_{m_{(i)}}, x_{n_{(i)}-1}\right)<\varepsilon
$$

By using triangular inequality, we have

$$
d\left(x_{m_{(i)}}, x_{n_{(i)}}\right) \leq d\left(x_{m_{(i)}}, x_{n_{(i)}-1}\right)+d\left(x_{n_{(i)}-1}, x_{n_{(i)}}\right) .
$$

By combining (2.5), (2.6), and (2.7) we obtain

$$
\varepsilon \leq d\left(x_{m_{(i)}}, x_{n_{(i)}}\right)<d\left(x_{n_{(i)}-1}, x_{n_{(i)}}\right)+\varepsilon,
$$

taking $i \rightarrow \infty$, we get

$$
\lim _{i \rightarrow \infty} d\left(x_{m_{(i)}}, x_{n_{(i)}}\right)=\varepsilon .
$$

Also, we have

$$
d\left(x_{m_{(i)}}, x_{n_{(i)}-1}\right) \leq d\left(x_{m_{(i)}}, x_{n_{(i)}}\right)+d\left(x_{n_{(i)}}, x_{n_{(i)}-1}\right) .
$$

By taking $i \rightarrow \infty$ in (2.7) and (2.9), we obtain

$$
\varepsilon \leq \lim _{i \rightarrow \infty} d\left(x_{m_{(i)}}, x_{n_{(i)}-1}\right) \leq \varepsilon .
$$

Therefore,

$$
\lim _{i \rightarrow \infty} d\left(x_{m_{(i)}}, x_{n_{(i)}-1}\right)=\varepsilon
$$

Similarly we can show that

$$
\lim _{i \rightarrow \infty} d\left(x_{m_{(i)}+1}, x_{n_{(i)}}\right)=\varepsilon .
$$


From (2.1) and condition (1), since $x_{m_{(i)}} \asymp x_{n_{(i)}-1}$ we have

$$
\begin{aligned}
\psi\left(d\left(x_{m_{(i)}+1}, x_{n_{(i)}}\right)\right) & =\psi\left(d\left(f\left(x_{m_{(i)}}\right), f\left(x_{n_{(i)}-1}\right)\right)\right) \\
& \leq \theta\left(d\left(x_{m_{(i)}}, x_{n_{(i)}-1}\right)\right)-\varphi\left(d\left(x_{m_{(i)}}, x_{n_{(i)}-1}\right)\right) .
\end{aligned}
$$

By using the properties of $\psi$ as altering distance and from (2.11) we deduce that

$$
\lim _{i \rightarrow \infty} \psi\left(d\left(x_{m_{(i)}+1}, x_{n_{(i)}}\right)\right)=\psi(\varepsilon)
$$

Now take $i \rightarrow \infty$ in (2.12); we get

$$
\psi(\varepsilon) \leq \theta(\varepsilon)-\varphi(\varepsilon)
$$

Thus $\psi(\varepsilon)-\theta(\varepsilon)+\varphi(\varepsilon) \leq 0$, this is a contradiction due to $\varepsilon>0$. Therefore, $\left\{x_{n}\right\}$ is a Cauchy sequence. Since $X$ is complete, $\left\{x_{n}\right\}$ converges. Thus there exists $x^{*} \in X$ such that

$$
\lim _{n \rightarrow \infty} x_{n}=\lim _{n \rightarrow \infty} f\left(x_{n}\right)=x^{*}
$$

Now suppose that part (a) of condition (4) holds, i.e., $f$ is continuous. Then $x^{*}=$ $\lim _{n \rightarrow \infty} x_{n}=\lim _{n \rightarrow \infty} f\left(x_{n}\right)=f\left(x^{*}\right)$. Therefore, $x^{*}$ is a fixed point of $f$.

We suppose that (b) holds. Since $x_{n} \rightarrow x^{*}, x_{n} \asymp x^{*}$. By using condition (1) we obtain

$$
\psi\left(d\left(f\left(x^{*}\right), f\left(x_{n}\right)\right)\right) \leq \theta\left(d\left(x^{*}, x_{n}\right)\right)-\varphi\left(d\left(x^{*}, x_{n}\right)\right)
$$

Let $n \rightarrow \infty$. We have

$$
\psi\left(d\left(f\left(x^{*}\right), x^{*}\right)\right) \leq \theta\left(d\left(x^{*}, x^{*}\right)\right)-\varphi\left(d\left(x^{*}, x^{*}\right)\right)=0
$$

Thus $\psi\left(d\left(f\left(x^{*}\right), x^{*}\right)\right)=0$, i.e., $f\left(x^{*}\right)=x^{*}$.

Finally, we prove the uniqueness of the fixed point. Suppose $f$ has another fixed point $y^{*}$. From the assumption, there exists $z \in X$ such that $x^{*} \asymp z$ and $y^{*} \asymp z$. If $z=x^{*}$ or $z=y^{*}$, it is trivial. We suppose $z \neq x^{*}$ and $z \neq y^{*}$. Put $z_{0}=z$ and choose $z_{1} \in X$ such that $z_{1}=f\left(z_{0}\right)$. Then we have $z_{0} \asymp x^{*}$. By using condition (3) we deduce that $f\left(z_{0}\right) \asymp f\left(x^{*}\right)$, that is, $z_{1} \asymp x^{*}$; again use condition (3) and we have $f\left(z_{1}\right) \asymp f\left(x^{*}\right)$. Proceeding by induction, we obtain $z_{n} \asymp x^{*}$, for definiteness we assume $x^{*} \neq z_{n}$ for all $n \in \mathbb{N}$. Similarly, we have $y^{*} \asymp z_{n}$ and $y^{*} \neq z_{n}$ for all $n \in \mathbb{N}$. Now, by using condition (1) we deduce

$$
\begin{aligned}
\psi\left(d\left(x^{*}, z_{n}\right)\right) & =\psi\left(d\left(f\left(x^{*}\right), f\left(z_{n-1}\right)\right)\right) \\
& \leq \theta\left(d\left(x^{*}, z_{n-1}\right)\right)-\varphi\left(d\left(x^{*}, z_{n-1}\right)\right)
\end{aligned}
$$

Since

$$
\frac{\theta\left(d\left(x^{*}, z_{n-1}\right)\right)-\varphi\left(d\left(x^{*}, z_{n-1}\right)\right)}{\psi\left(d\left(x^{*}, z_{n-1}\right)\right)}<1,
$$

we have

$$
\psi\left(d\left(x^{*}, z_{n}\right)\right)<\psi\left(d\left(x^{*}, z_{n-1}\right)\right) .
$$


Due to $\psi$ being an altering distance, we find that $\left\{d\left(x^{*}, z_{n}\right)\right\}$ is a decreasing sequence. Consequently, there exists a positive number $\ell$ such that $d\left(x^{*}, z_{n}\right) \rightarrow \ell$. Let $n \rightarrow \infty$ in (2.13), and we obtain

$$
\psi(\ell) \leq \theta(\ell)-\varphi(\ell)
$$

If $\ell \neq 0$, then $\psi(\ell)-\theta(\ell)+\varphi(\ell) \leq 0$, a contradiction. Thus $\ell=0$, i.e., $d\left(x^{*}, z_{n}\right) \rightarrow 0$. Similarly, we can deduce that $d\left(y^{*}, z_{n}\right) \rightarrow 0$. In the other hand, we have $d\left(x^{*}, y^{*}\right) \leq d\left(x^{*}, z_{n}\right)+$ $d\left(z_{n}, y^{*}\right)$. We get $x^{*}=y^{*}$ when $n \rightarrow \infty$. Hence, the fixed point of $f$ is unique.

Note that condition (1) in the above theorem is also used in [20].

If we take $\theta=\psi$ in Theorem 2.5, we obtain the following corollary.

Corollary 2.6 Let $(X, d, \preceq)$ be a complete partially ordered metric space. Let $f: X \rightarrow X$ be a mapping which obeys the following conditions:

(1) there exist an altering distance function $\psi$ and a lower semi-continuous function $\varphi:[0, \infty) \rightarrow[0, \infty)$ such that

$$
\psi(d(f(x), f(y))) \leq \psi(d(x, y))-\varphi(d(x, y)) \quad \text { for } x \succeq y,
$$

where $\varphi(0)=0$;

(2) there exists $x_{0} \in X$ such that $x_{0} \asymp f\left(x_{0}\right)$;

(3) $f$ is nondecreasing;

(4) (a) $f$ is continuous or

(b) if $x_{n} \rightarrow x$ when $n \rightarrow \infty$ in $X$, then $x_{n} \asymp x$ for all $n$.

Then $f$ has a fixed point. Moreover, iffor each $x, y \in X$ there exists $z \in X$ which is comparable to $x$ and $y$, then the fixed point is unique.

If we consider the special functions $\psi(t)=t$ and $\varphi(t)=(1-k) t$ where $k \in[0,1)$ and by using the above corollary, we can easily generalize the results of Ran and Reurings [9] and Nieto and Rodríguez-López [15] as follows.

Corollary 2.7 Let $(X, d, \preceq)$ be a complete partially ordered metric space and let $f: X \rightarrow X$ be a mapping which obeys the following conditions:

(1) there exists a constant $k \in[0,1)$ such that

$$
d(f(x), f(y)) \leq k d(x, y) \quad \text { for } x \geq y
$$

(2) there exist $x_{0} \in X$ such that $x_{0} \asymp f\left(x_{0}\right)$;

(3) $f$ is nondecreasing;

(4) (a) $f$ is continuous or

(b) if $x_{n} \rightarrow x$ when $n \rightarrow \infty$ in $X$, then $x_{n} \asymp x$ for all $n$.

Then $f$ has a fixed point. Moreover, iffor each $x, y \in X$ there exists $z \in X$ which is comparable to $x$ and $y$, then the fixed point is unique.

\section{Coupled fixed point theorems}

In this section, we obtain some coupled fixed point results. The notions of a coupled fixed point and the mixed monotone property in metric spaces endowed with a partial order were introduced by Bhaskar and Lakshmikantham in [10] as follows. 
Definition 3.1 Let $X$ be a nonempty set and $F: X \times X \rightarrow X$ be a mapping. An element $(x, y) \in X \times X$, is called a coupled fixed point of $F$ if

$$
x=F(x, y) \text { and } y=F(y, x) .
$$

Definition 3.2 Let $(X, \preceq)$ be a partially ordered set and let $F: X \times X \rightarrow X$ be a mapping. We say that the mapping $F$ has the mixed monotone property if $F$ is monotone nondecreasing in its first argument and is monotone nonincreasing in its second argument. That is, for any $x, y \in X$,

$$
x_{1}, x_{2} \in X, \quad x_{1} \preceq x_{2} \quad \Rightarrow \quad F\left(x_{1}, y\right) \preceq F\left(x_{2}, y\right)
$$

and

$$
y_{1}, y_{2} \in X, \quad y_{1} \preceq y_{2} \quad \Rightarrow \quad F\left(x, y_{1}\right) \succeq F\left(x, y_{2}\right) .
$$

The following theorems are the main theoretical results of Bhaskar and Lakshmikantham [10].

Theorem 3.3 Let $(X, d, \preceq)$ be a complete partially ordered metric space and let $F: X \times$ $X \rightarrow X$ be a continuous mapping having the mixed monotone property on $X$. Assume that there exists a constant $k \in[0,1)$ with

$$
d(F(x, y), F(u, v)) \leq \frac{k}{2}[d(x, u)+d(y, v)] \quad \text { for each } x \succeq u, y \preceq v .
$$

If there exist $x_{0}, y_{0} \in X$ such that $x_{0} \preceq F\left(x_{0}, y_{0}\right)$ and $y_{0} \succeq F\left(y_{0}, x_{0}\right)$, then there exist $x^{*}, y^{*} \in X$ such that

$$
x^{*}=F\left(x^{*}, y^{*}\right) \text { and } y^{*}=F\left(y^{*}, x^{*}\right) .
$$

Theorem 3.4 Let $(X, d, \preceq)$ be a complete partially ordered metric space. Assume that $X$ has the following properties:

(1) if a nondecreasing sequence $\left\{x_{n}\right\} \rightarrow x$, then $x_{n} \preceq x$, for all $n$;

(2) if a nonincreasing sequence $\left\{y_{n}\right\} \rightarrow y$, then $y_{n} \succeq y$, for all $n$.

Let $F: X \times X \rightarrow X$ be a mapping having the mixed monotone property on $X$. Assume that there exists a constant $k \in[0,1)$ with

$$
d(F(x, y), F(u, v)) \leq \frac{k}{2}[d(x, u)+d(y, v)] \quad \text { for each } x \geq u, y \preceq v .
$$

If there exist $x_{0}, y_{0} \in X$ such that $x_{0} \preceq F\left(x_{0}, y_{0}\right)$ and $y_{0} \succeq F\left(y_{0}, x_{0}\right)$, then there exist $x^{*}, y^{*} \in X$ such that

$$
x^{*}=F\left(x^{*}, y^{*}\right) \text { and } y^{*}=F\left(y^{*}, x^{*}\right) .
$$

In [21], Samet et al. defined the mappings $\eta, \delta: Y \times Y \rightarrow[0, \infty)$, where $Y=X \times X$ and $(X, \preceq)$ is a partially ordered set endowed with a metric $d$, by

$$
\eta((x, y),(u, v))=d(x, u)+d(y, v), \quad \delta((x, y),(u, v))=\max \{d(x, u), d(y, v)\}
$$

for all $(x, y),(u, v) \in Y$. 
It is easy to show that $(Y, \eta)$ and $(Y, \delta)$ are metric spaces. Let $F: Y \rightarrow X$ be a mapping and define $T: Y \rightarrow Y$ by

$$
T(x, y)=(F(x, y), F(y, x)) \quad \text { for all }(x, y) \in Y \text {. }
$$

Let $(X, \preceq)$ be a partially ordered set and $(x, y),(u, v) \in X \times X$. We say that $(x, y) \preceq_{2}(u, v)$ if $x \preceq u$ and $y \succeq v$.

Here we recall the following lemma.

Lemma 3.5 [21] The following properties hold:

(a) $(X, d)$ is complete if and only if $(Y, \eta)$ and $(Y, \delta)$ are complete;

(b) $F$ has the mixed monotone property if and only if $T$ is monotone nondecreasing with respect to $\preceq_{2}$;

(c) $(x, y) \in X \times X$ is a coupled fixed point of $F$ if and only if $(x, y)$ is a fixed point of $T$.

Now, we are ready to formulate our next result.

Theorem 3.6 Let $(X, d, \preceq)$ be a complete partially ordered metric space. Let $F: X \times X \rightarrow X$ be a mapping which obeys the following conditions:

(1) there exist an altering distance function $\psi$, an upper semi-continuous function $\theta:[0, \infty) \rightarrow[0, \infty)$, and a lower semi-continuous function $\varphi:[0, \infty) \rightarrow[0, \infty)$ such that for all $(x, y),(u, v) \in X \times X$ with $(x, y) \preceq_{2}(u, v)$

$$
\psi(d(F(x, y), F(u, v))) \leq \theta(\max \{d(x, u), d(y, v)\})-\varphi(\max \{d(x, u), d(y, v)\}),
$$

where $\theta(0)=\varphi(0)=0$ and $\psi(t)-\theta(t)+\varphi(t)>0$ for all $t>0$;

(2) there exist $x_{0}, y_{0} \in X$ such that $x_{0} \preceq F\left(x_{0}, y_{0}\right)$ and $y_{0} \succeq F\left(y_{0}, x_{0}\right)$;

(3) F has the mixed monotone property;

(4) (a) $F$ is continuous or

(b) $X$ has the following properties:

(i) if a nondecreasing sequence $\left\{x_{n}\right\} \rightarrow x$, then $x_{n} \preceq x$ for all $n$;

(ii) if a nonincreasing sequence $\left\{y_{n}\right\} \rightarrow y$, then $y_{n} \succeq y$ for all $n$.

Then $F$ has a coupled fixed point $\left(x^{*}, y^{*}\right)$. Moreover, if for any $(x, y),(u, v) \in X \times X$ there exists $(w, z) \in X \times X$ such that $(x, y) \preceq_{2}(w, z)$ and $(u, v) \preceq_{2}(w, z)$, then $\left(x^{*}, x^{*}\right)$ is the unique coupled fixed point of $F$.

Proof We prove the theorem in three steps.

Step 1. We show that the function $T$ which is defined in (3.1) satisfies condition (1) in Theorem 2.5. By using condition (1) we get

$$
\psi(d(F(x, y), F(u, v))) \leq \theta(\max \{d(x, u), d(y, v)\})-\varphi(\max \{d(x, u), d(y, v)\})
$$

and

$$
\begin{aligned}
\psi(d(F(v, u), F(y, x))) & =\psi(d(F(y, x), F(v, u))) \\
& \leq \theta(\max \{d(x, u), d(y, v)\})-\varphi(\max \{d(x, u), d(y, v)\})
\end{aligned}
$$


for each $(x, y),(u, v) \in X \times X$ with $(x, y) \preceq_{2}(u, v)$. Thus

$$
\begin{aligned}
& \max \{\psi(d(F(x, y), F(u, v))), \psi(d(F(y, x), F(v, u)))\} \\
& \quad \leq \theta(\max \{d(x, u), d(y, v)\})-\varphi(\max \{d(x, u), d(y, v)\}) .
\end{aligned}
$$

Since $\psi$ is nondecreasing, we have $\psi(\max (a, b))=\max (\psi(a), \psi(b))$ for $a, b \in[0, \infty)$. Therefore,

$$
\begin{aligned}
& \psi(\max \{d(F(x, y), F(u, v)), d(F(y, x), F(v, u))\}) \\
& \quad \leq \theta(\max \{d(x, u), d(y, v)\})-\varphi(\max \{d(x, u), d(y, v)\}) .
\end{aligned}
$$

By using the definition of $\delta$ we have

$$
\psi(\delta((F(x, y), F(y, x)),(F(u, v), F(v, u)))) \leq \theta(\delta((x, y),(u, v)))-\varphi(\delta((x, y),(u, v)))
$$

and by using the definition of $T$ we obtain

$$
\psi(\delta(T(x, y), T(u, v))) \leq \theta(\delta((x, y),(u, v)))-\varphi(\delta((x, y),(u, v)))
$$

for all $(x, y),(u, v) \in Y$ with $(x, y) \preceq_{2}(u, v)$.

Step 2. Let us prove that $F$ has a coupled fixed point. We have $(X, d)$ is complete. Then, by Lemma $3.5,(Y, \delta)$ is also complete. By using condition (2) there exist $x_{0}, y_{0} \in X$ such that $x_{0} \preceq F\left(x_{0}, y_{0}\right)$ and $y_{0} \succeq F\left(y_{0}, x_{0}\right)$, so we deduce that

$$
\left(x_{0}, y_{0}\right) \preceq_{2}\left(F\left(x_{0}, y_{0}\right), F\left(y_{0}, x_{0}\right)\right) \text {, }
$$

thus,

$$
\left(x_{0}, y_{0}\right) \preceq_{2} T\left(x_{0}, y_{0}\right) .
$$

In condition (3) $F$ has the mixed monotone property so by using Lemma 3.5 we find that $T$ is monotone nondecreasing with respect to $\preceq_{2}$.

Now, from condition (4)(a) since $f$ is continuous, $T$ is continuous. Also, in case (b), if (i) and (ii) are satisfied, then we see that if a nondecreasing sequence $\left\{\left(x_{n}, y_{n}\right)\right\}$ with respect to $\preceq_{2}$ in $Y$ converges to $(x, y)$, then $\left(x_{n}, y_{n}\right) \preceq_{2}(x, y)$ for all $n$. Consequently, $T$ satisfies all the conditions of Theorem 2.5. Hence, $T$ has a fixed point, which leads to $F$ having a coupled fixed point.

Step 3. Let us now show the uniqueness of coupled fixed point and it has the form $\left(x^{*}, x^{*}\right)$. We suppose that $\left(x^{*}, y^{*}\right)$ is a coupled fixed point of $F$ and we take $(u, v) \in X \times X$ is another coupled fixed point of $F$ then there exists $(w, z) \in X \times X$ such that $\left(x^{*}, y^{*}\right) \preceq_{2}(w, z)$ and $(u, v) \preceq_{2}(w, z)$. Therefore, $\left(x^{*}, y^{*}\right)$ and $(u, v)$ are fixed points of $T$ with $\left(x^{*}, y^{*}\right) \preceq_{2}(w, z)$ and $(u, v) \preceq_{2}(w, z)$. From the last part of Theorem 2.5 we find that $T$ has a unique fixed point, which leads to the uniqueness of the coupled fixed point of $F$. Now, since $\left(x^{*}, y^{*}\right)$ is the coupled fixed point of $F$, that is, $x^{*}=F\left(x^{*}, y^{*}\right)$ and $y^{*}=F\left(y^{*}, x^{*}\right)$, then $\left(y^{*}, x^{*}\right)$ is also a coupled fixed point of $F$. By the uniqueness of the coupled fixed point we obtain $\left(x^{*}, x^{*}\right)$ is the unique coupled fixed point of $F$. 
Theorem 3.7 Let $(X, d, \preceq)$ be a complete partially ordered metric space. Let $F: X \times X \rightarrow X$ be a mapping. Suppose the following conditions hold:

(1) there exist an altering distance function $\psi$, an upper semi-continuous function $\theta:[0, \infty) \rightarrow[0, \infty)$, and a lower semi-continuous function $\varphi:[0, \infty) \rightarrow[0, \infty)$ such that for all $(x, y),(u, v) \in X \times X$ with $(x, y) \preceq_{2}(u, v)$

$$
\psi(d(F(x, y), F(u, v))) \leq \frac{1}{2} \theta(d(x, u)+d(y, v))-\varphi\left(\frac{d(x, u)+d(y, v)}{2}\right),
$$

where $\varphi(0)=\theta(0)=0$ and $\psi(t)-\theta(t)+\varphi(t)>0$ for all $t>0$;

(2) $\psi(a+b) \leq \psi(a)+\psi(b)$ and $\varphi(c) \leq 2 \varphi(c / 2)$ for all $a, b, c \in[0, \infty)$;

(3) $F$ has the mixed monotone property;

(4) there exist $x_{0}, y_{0} \in X$ such that $x_{0} \preceq F\left(x_{0}, y_{0}\right)$ and $y_{0} \succeq F\left(y_{0}, x_{0}\right)$;

(5) (a) $F$ is continuous or

(b) $X$ has the following properties:

(i) if a nondecreasing sequence $\left\{x_{n}\right\} \rightarrow x$, then $x_{n} \preceq x$ for all $n$;

(ii) if a nonincreasing sequence $\left\{y_{n}\right\} \rightarrow y$, then $y_{n} \succeq y$ for all $n$.

Then $F$ has a coupled fixed point $\left(x^{*}, y^{*}\right)$. Moreover, if for any $(x, y),(u, v) \in X \times X$ there exists $(w, z) \in X \times X$ such that $(x, y) \preceq_{2}(w, z)$ and $(u, v) \preceq_{2}(w, z)$, then $\left(x^{*}, x^{*}\right)$ is the unique coupled fixed point of $F$.

Proof The main object in this proof is to show that the function $T$ which is defined in (3.1) satisfies condition (1) in Theorem 2.5. By using condition (1), we have

$$
\psi(d(F(x, y), F(u, v))) \leq \frac{1}{2} \theta(d(x, u)+d(y, v))-\varphi\left(\frac{d(x, u)+d(y, v)}{2}\right)
$$

and

$$
\psi(d(F(y, x), F(v, u))) \leq \frac{1}{2} \theta(d(x, u)+d(y, v))-\varphi\left(\frac{d(x, u)+d(y, v)}{2}\right)
$$

for $(x, y) \preceq_{2}(u, v)$. By summing the above inequalities, we obtain

$$
\begin{aligned}
& \psi(d(F(x, y), F(u, v)))+\psi(d(F(y, x), F(v, u))) \\
& \quad \leq \theta(d(x, u)+d(y, v))-2 \varphi\left(\frac{d(x, u)+d(y, v)}{2}\right) .
\end{aligned}
$$

When we utilize condition (2), we get

$$
\psi(d(F(x, y), F(u, v))+d(F(y, x), F(v, u))) \leq \theta(d(x, u)+d(y, v))-\varphi(d(x, u)+d(y, v)) .
$$

Now, we use the definition of $\eta$,

$$
\psi(\eta((F(x, y), F(y, x)),(F(u, v), F(v, u)))) \leq \theta(\eta((x, y),(u, v)))-\varphi(\eta((x, y),(u, v)))
$$

and by using the definition of $T$ we obtain

$$
\psi(\eta(T(x, y), T(u, v))) \leq \theta(\eta((x, y),(u, v)))-\varphi(\eta((x, y),(u, v)))
$$


for all $(x, y),(u, v) \in Y$ with $(x, y) \preceq_{2}(u, v)$. The rest of the proof follows exactly the proof of Theorem 3.6.

Remark 3.8 The results of Harjani et al. [16] and Luong and Thuan [17] are extended and generalized by Theorem 3.6 and Theorem 3.7, respectively.

Note that the following theorem can be proved the exactly same manner as above and the motivation of this theorem will be known in the succeeding section.

Theorem 3.9 Let $(X, d, \preceq)$ be a complete partially ordered metric space. Let $F: X \times X \rightarrow X$ be a mapping. Suppose the following conditions hold:

(1) there exist an altering distance function $\psi$, an upper semi-continuous function $\theta:[0, \infty) \rightarrow[0, \infty)$, and a lower semi-continuous function $\varphi:[0, \infty) \rightarrow[0, \infty)$ such that for all $(x, y),(u, v) \in X \times X$ with $(x, y) \preceq_{2}(u, v)$

$$
\psi(d(F(x, y), F(u, v))) \leq \theta\left(\frac{d(x, u)+d(y, v)}{2}\right)-\frac{1}{2} \varphi(d(x, u)+d(y, v)),
$$

where $\varphi(0)=\theta(0)=0$ and $\psi(t)-\theta(t)+\varphi(t)>0$ for all $t>0$;

(2) $\psi(a+b) \leq \psi(a)+\psi(b)$ and $2 \theta(c / 2) \leq \theta(c)$ for all $a, b, c \in[0, \infty)$;

(3) $F$ has the mixed monotone property;

(4) there exist $x_{0}, y_{0} \in X$ such that $x_{0} \preceq F\left(x_{0}, y_{0}\right)$ and $y_{0} \succeq F\left(y_{0}, x_{0}\right)$;

(5) (a) $F$ is continuous or

(b) $X$ has the following properties:

(i) if a nondecreasing sequence $\left\{x_{n}\right\} \rightarrow x$, then $x_{n} \preceq x$ for all $n$;

(ii) if a nonincreasing sequence $\left\{y_{n}\right\} \rightarrow y$, then $y_{n} \succeq y$ for all $n$.

Then $F$ has a coupled fixed point $\left(x^{*}, y^{*}\right)$. Moreover, if for any $(x, y),(u, v) \in X \times X$ there exists $(w, z) \in X \times X$ such that $(x, y) \preceq_{2}(w, z)$ and $(u, v) \preceq_{2}(w, z)$, then $\left(x^{*}, x^{*}\right)$ is the unique coupled fixed point of $F$.

The following theorem was proved by Bhaskar and Lakshmikantham in [10]. Now we will prove it by a different way.

Theorem 3.10 Let $(X, d, \preceq)$ be a complete partially ordered metric space and let $F: X \times$ $X \rightarrow X$ be a mapping which obeys the following conditions:

(1) there exists a constant $k \in[0,1)$ such that for all $(x, y),(u, v) \in X \times X$ with $(x, y) \preceq_{2}(u, v)$

$$
d(F(x, y), F(u, v)) \leq \frac{k}{2}[d(x, u)+d(y, v)]
$$

(2) there exist $x_{0}, y_{0} \in X$ such that $x_{0} \preceq F\left(x_{0}, y_{0}\right)$ and $y_{0} \succeq F\left(y_{0}, x_{0}\right)$;

(3) $F$ has the mixed monotone property;

(4) (a) $F$ is continuous or

(b) $X$ has the following properties:

(i) if a nondecreasing sequence $\left\{x_{n}\right\} \rightarrow x$, then $x_{n} \preceq x$ for all $n$;

(ii) if a nonincreasing sequence $\left\{y_{n}\right\} \rightarrow y$, then $y_{n} \succeq y$ for all $n$.

Then $F$ has a coupled fixed point $\left(x^{*}, y^{*}\right)$. Moreover, if for any $(x, y),(u, v) \in X \times X$ there exists $(w, z) \in X \times X$ such that $(x, y) \preceq_{2}(w, z)$ and $(u, v) \preceq_{2}(w, z)$, then $\left(x^{*}, x^{*}\right)$ is the unique coupled fixed point of $F$. 
Proof Our major work in this proof is to show that the function $T$ which is defined in (3.1) satisfies condition (1) in Corollary 2.7. By using condition (1) we have

$$
d(F(x, y), F(u, v)) \leq \frac{k}{2}[d(x, u)+d(y, v)]
$$

and

$$
d(F(y, x), F(v, u)) \leq \frac{k}{2}[d(x, u)+d(y, v)]
$$

for $(x, y) \preceq_{2}(u, v)$. Hence

$$
d(F(x, y), F(u, v))+d(F(y, x), F(v, u)) \leq k[d(x, u)+d(y, v)] .
$$

By using the definition of $\eta$, we have

$$
\eta((F(x, y), F(y, x)),(F(u, v), F(v, u))) \leq k \eta((x, y),(u, v)),
$$

and by utilizing the definition of $T$, we obtain

$$
\eta(T(x, y), T(u, v)) \leq k \eta((x, y),(u, v))
$$

for all $(x, y),(u, v) \in Y$ with $(x, y) \preceq_{2}(u, v)$. The rest of the proof follows the proof of Theorem 3.6 and then we apply Corollary 2.7.

\section{Coupled coincidence point without compatibility}

In this section, we get new coupled coincidence point results. The notions of coupled coincidence point and mixed $g$-monotone property have been established recently in the work [11] by Lakshmikantham and Ćirić. These concepts are defined as follows.

Definition 4.1 Let $X$ be a nonempty set and let $F: X \times X \rightarrow X$ and $g: X \rightarrow X$ be mappings. We say that $(x, y) \in X \times X$ is a coupled coincidence point of $F$ and $g$ if $F(x, y)=g(x)$ and $F(y, x)=g(y)$ for $x, y \in X$.

Definition 4.2 Let $(X, \preceq)$ be a partially ordered set and let $F: X \times X \rightarrow X$ and $g: X \rightarrow$ $X$ be mappings. We say $F$ has the mixed $g$-monotone property if $F$ is monotone $g$-nondecreasing in its first argument and is monotone $g$-nonincreasing in its second argument. That is, for any $x, y \in X$,

$$
x_{1}, x_{2} \in X, \quad g\left(x_{1}\right) \preceq g\left(x_{2}\right) \quad \Rightarrow \quad F\left(x_{1}, y\right) \preceq F\left(x_{2}, y\right)
$$

and

$$
y_{1}, y_{2} \in X, \quad g\left(y_{1}\right) \preceq g\left(y_{2}\right) \quad \Rightarrow \quad F\left(x, y_{1}\right) \succeq F\left(x, y_{2}\right) .
$$

Recently, the notion of compatibility of $F$ and $g$ was defined by Choudhury and Kundu [12] as follows. 
Definition 4.3 Let $(X, d)$ be a metric space and let $g: X \rightarrow X, F: X \times X \rightarrow X$. The mappings $g$ and $F$ are compatible if

$$
\lim _{n \rightarrow \infty} d\left(g\left(F\left(x_{n}, y_{n}\right)\right), F\left(g\left(x_{n}\right), g\left(y_{n}\right)\right)\right)=0
$$

and

$$
\lim _{n \rightarrow \infty} d\left(g\left(F\left(y_{n}, x_{n}\right)\right), F\left(g\left(y_{n}\right), g\left(x_{n}\right)\right)\right)=0
$$

whenever $\left\{x_{n}\right\}$ and $\left\{y_{n}\right\}$ are sequences in $X$, such that $\lim _{n \rightarrow \infty} F\left(x_{n}, y_{n}\right)=\lim _{n \rightarrow \infty} g\left(x_{n}\right)=x$ and $\lim _{n \rightarrow \infty} F\left(y_{n}, x_{n}\right)=\lim _{n \rightarrow \infty} g\left(y_{n}\right)=y$, for all $x, y \in X$ are satisfied.

Now, we are in the position to prove our result in coupled coincidence point which is a generalization of the main results of Razani and Parvaneh [19] and Alsulami [13].

Theorem 4.4 Let $(X, d, \preceq)$ be a partially ordered metric space. Let the mappings $g: X \rightarrow X$ and $F: X \times X \rightarrow X$ have the following conditions:

(1) $g(X)$ is complete, $g$ is continuous and increasing;

(2) $F(X \times X) \subset g(X)$;

(3) there exist an altering distance function $\psi$, an upper semi-continuous function $\theta:[0, \infty) \rightarrow[0, \infty)$, and a lower semi-continuous function $\varphi:[0, \infty) \rightarrow[0, \infty)$ such that for all $x, y, u, v \in X$ with $(g(x), g(y)) \preceq_{2}(g(u), g(v))$ we have

$$
\begin{aligned}
\psi(d(F(x, y), F(u, v))) \leq & \theta(\max \{d(g(x), g(u)), d(g(y), g(v))\}) \\
& -\varphi(\max \{d(g(x), g(u)), d(g(y), g(v))\}),
\end{aligned}
$$

where $\varphi(0)=\theta(0)=0$ and $\psi(t)-\theta(t)+\varphi(t)>0$ for all $t>0$;

(4) there exist $x_{0}, y_{0} \in X$ such that $g\left(x_{0}\right) \preceq F\left(x_{0}, y_{0}\right)$ and $g\left(y_{0}\right) \succeq F\left(y_{0}, x_{0}\right)$;

(5) $F$ has the mixed g-monotone property;

(6) (a) $F$ is continuous or

(b) $X$ has the following properties:

(i) if a nondecreasing sequence $\left\{x_{n}\right\} \rightarrow x$, then $x_{n} \preceq x$ for all $n$,

(ii) if a nonincreasing sequence $\left\{y_{n}\right\} \rightarrow y$, then $y_{n} \succeq y$ for all $n$.

Then $g$ and $F$ have a coupled coincidence point. Moreover, if for any $(x, y),(u, v) \in X \times X$ there exists $(w, z) \in X \times X$ such that $(x, y) \preceq_{2}(w, z)$ and $(u, v) \preceq_{2}(w, z)$, then $(s, s)$ is the unique coupled coincidence point of $F$ and $g$.

Proof We define a map $G: g(X) \times g(X) \rightarrow g(X)$ by

$$
G(g(x), g(y))=F(x, y)
$$

for all $g(x), g(y) \in g(X)$. G is well defined on $g(X)$ since $g$ is increasing. First, we show that the map $G: g(X) \times g(X) \rightarrow g(X)$ satisfies all the assumptions of Theorem 3.6. It is clear $(g(X), d, \preceq)$ is a partially ordered metric space. From condition (3) and (4.1), we have

$$
\begin{aligned}
\psi(d(G(g(x), g(y)), G(g(u), g(v)))) \leq & \theta(\max \{d(g(x), g(u)), d(g(y), g(v))\}) \\
& -\varphi(\max \{d(g(x), g(u)), d(g(y), g(v))\})
\end{aligned}
$$


for all $g(x), g(y), g(u), g(v) \in g(X)$ with $(g(x), g(y)) \preceq_{2}(g(u), g(v))$. From condition (4), there exist $g\left(x_{0}\right), g\left(y_{0}\right) \in g(X)$ such that

$$
g\left(x_{0}\right) \preceq G\left(g\left(x_{0}\right), g\left(y_{0}\right)\right) \quad \text { and } \quad g\left(y_{0}\right) \succeq G\left(g\left(y_{0}\right), g\left(x_{0}\right)\right) .
$$

From condition (5),

$$
\text { if }(g(x), g(y)) \preceq_{2}(g(u), g(v)) \text { then } G(g(x), g(y)) \preceq G(g(u), g(v))
$$

for all $g(x), g(y), g(u), g(v) \in g(X)$. Thus, $G$ has the mixed monotone property. By using part (a) of condition (6), $G$ is continuous since $F$ is continuous. Moreover, we suppose that the assumption (b) holds. Since $g$ is continuous and increasing, $g(X)$ also has the following properties:

(i') if a nondecreasing sequence $\left\{g\left(x_{n}\right)\right\} \rightarrow g(x)$, then $g\left(x_{n}\right) \preceq g(x)$ for all $n$,

(ii') if a nonincreasing sequence $\left\{g\left(y_{n}\right)\right\} \rightarrow g(y)$, then $g\left(y_{n}\right) \succeq g(y)$ for all $n$.

Moreover, due to $g$ is increasing, we see that for any $(g(x), g(y)),(g(u), g(v)) \in g(X) \times g(X)$ there exists $(g(w), g(z)) \in g(X) \times g(X)$ such that $(g(x), g(y)) \preceq_{2}(g(w), g(z))$ and $(g(u), g(v)) \preceq_{2}$ $(g(w), g(z))$. Therefore, all the assumptions of Theorem 3.6 are satisfied. Hence, $G$ has a unique coupled fixed point $\left(x^{*}, x^{*}\right) \in g(X) \times g(X)$.

Now, we prove that $F$ and $g$ have a unique coupled coincidence point. Let $\left(x^{*}, x^{*}\right)$ be the coupled fixed point of $G$, that is, $x^{*}=G\left(x^{*}, x^{*}\right)$. Since $x^{*} \in g(X)$ and $g$ is increasing, there exists a unique $s \in X$ such that $g(s)=x^{*}$. Thus, $g(s)=G(g(s), g(s))$, i.e., $g(s)=F(s, s)$. Therefore, $(s, s)$ is a unique coupled coincidence point of $F$ and $g$.

If we take $\psi(t)=\theta(t)=t$ and $\varphi(t)=(1-k) t$ with $k<1$ in Theorem 4.4, we have the following corollary.

Corollary 4.5 Let $(X, d, \preceq)$ be a complete partially ordered metric space. Let the mappings $g: X \rightarrow X$ and $F: X \times X \rightarrow X$ obey the following conditions:

(1) $g(X)$ is complete, $g$ is continuous and increasing;

(2) $F(X \times X) \subset g(X)$;

(3) there exists $k \in[0,1)$ such that for all $x, y, u, v \in X$ with $(g(x), g(y)) \preceq_{2}(g(u), g(v))$ we have

$$
d(F(x, y), F(u, v)) \leq k \max \{d(g(x), g(u)), d(g(y), g(v))\}
$$

(4) there exist $x_{0}, y_{0} \in X$ such that $g\left(x_{0}\right) \preceq F\left(x_{0}, y_{0}\right)$ and $g\left(y_{0}\right) \succeq F\left(y_{0}, x_{0}\right)$;

(5) $F$ has the mixed g-monotone property;

(6) (a) F is continuous or

(b) $X$ has the following properties:

(i) if a nondecreasing sequence $\left\{x_{n}\right\} \rightarrow x$, then $x_{n} \preceq x$ for all $n$,

(ii) if a nonincreasing sequence $\left\{y_{n}\right\} \rightarrow y$, then $y_{n} \geq y$ for all $n$.

Then $g$ and $F$ have a coupled coincidence point. Moreover, if for any $(x, y),(u, v) \in X \times X$ there exists $(w, z) \in X \times X$ such that $(x, y) \preceq_{2}(w, z)$ and $(u, v) \preceq_{2}(w, z)$, then $(s, s)$ is the unique coupled coincidence point of $F$ and $g$. 
The following result is an extension of the main result of Alotaibi and Alsulami [18] without using the compatibility.

Theorem 4.6 Let $(X, d, \preceq)$ be a partially ordered metric space. Let the mappings $g: X \rightarrow X$ and $F: X \times X \rightarrow X$ obey the following conditions:

(1) $g(X)$ is complete, $g$ is continuous and increasing;

(2) $F(X \times X) \subset g(X)$;

(3) there exist an altering distance function $\psi$, an upper semi-continuous function $\theta:[0, \infty) \rightarrow[0, \infty)$, and a lower semi-continuous function $\varphi:[0, \infty) \rightarrow[0, \infty)$ such that for all $x, y, u, v \in X$ with $(g(x), g(y)) \preceq_{2}(g(u), g(v))$

$$
\begin{aligned}
\psi(d(F(x, y), F(u, v))) \leq & \frac{1}{2} \theta(d(g(x), g(u))+d(g(y), g(v))) \\
& -\varphi\left(\frac{d(g(x), g(u))+d(g(y), g(v))}{2}\right)
\end{aligned}
$$

where $\varphi(0)=\theta(0)=0$ and $\psi(t)-\theta(t)+\varphi(t)>0$ for all $t>0$;

(4) $\psi(a+b) \leq \psi(a)+\psi(b)$ and $\varphi(c) \leq 2 \varphi(c / 2)$ for all $a, b, c \in[0, \infty)$;

(5) there exist $x_{0}, y_{0} \in X$ such that $g\left(x_{0}\right) \preceq F\left(x_{0}, y_{0}\right)$ and $g\left(y_{0}\right) \succeq F\left(y_{0}, x_{0}\right)$;

(6) $F$ has the mixed $g$-monotone property;

(7) (a) $F$ is continuous or

(b) $X$ has the following properties:

(i) if a nondecreasing sequence $\left\{x_{n}\right\} \rightarrow x$, then $x_{n} \preceq x$ for all $n$,

(ii) if a nonincreasing sequence $\left\{y_{n}\right\} \rightarrow y$, then $y_{n} \succeq y$ for all $n$.

Then $g$ and $F$ have a coupled coincidence point. Moreover, if for any $(x, y),(u, v) \in X \times X$ there exists $(w, z) \in X \times X$ such that $(x, y) \preceq_{2}(w, z)$ and $(u, v) \preceq_{2}(w, z)$, then $(s, s)$ is the unique coupled coincidence point of $F$ and $g$.

Proof Let $G: g(X) \times g(X) \rightarrow g(X)$ be the map which is defined in (4.1). From condition (3), we have

$$
\begin{aligned}
\psi(d(G(g(x), g(y)), G(g(u), g(v)))) \leq & \frac{1}{2} \theta(d(g(x), g(u))+d(g(y), g(v))) \\
& -\varphi\left(\frac{d(g(x), g(u))+d(g(y), g(v))}{2}\right)
\end{aligned}
$$

for all $g(x), g(y), g(u), g(v) \in g(X)$ with $(g(x), g(y)) \preceq_{2}(g(u), g(v))$. The rest of the proof is similar to the proof of Theorem 4.4 and by applying Theorem 3.7 we are done.

Applying Theorem 3.9 and Theorem 4.6 the following theorem can be proved easily.

Theorem 4.7 Let $(X, d, \preceq)$ be a partially ordered metric space. Let the mappings $g: X \rightarrow X$ and $F: X \times X \rightarrow X$ obey the following conditions:

(1) $g(X)$ is complete, $g$ is continuous and increasing;

(2) $F(X \times X) \subset g(X)$;

(3) there exist an altering distance function $\psi$, an upper semi-continuous function $\theta:[0, \infty) \rightarrow[0, \infty)$, and a lower semi-continuous function $\varphi:[0, \infty) \rightarrow[0, \infty)$ such 
that for all $x, y, u, v \in X$ with $(g(x), g(y)) \preceq_{2}(g(u), g(v))$

$$
\begin{aligned}
\psi(d(F(x, y), F(u, v))) \leq & \theta\left(\frac{d(g(x), g(u))+d(g(y), g(v))}{2}\right) \\
& -\frac{1}{2} \varphi(d(g(x), g(u))+d(g(y), g(v))),
\end{aligned}
$$

where $\varphi(0)=\theta(0)=0$ and $\psi(t)-\theta(t)+\varphi(t)>0$ for all $t>0$;

(4) $\psi(a+b) \leq \psi(a)+\psi(b)$ and $2 \theta(c / 2) \leq \theta(c)$ for all $a, b, c \in[0, \infty)$;

(5) there exist $x_{0}, y_{0} \in X$ such that $g\left(x_{0}\right) \preceq F\left(x_{0}, y_{0}\right)$ and $g\left(y_{0}\right) \succeq F\left(y_{0}, x_{0}\right)$;

(6) $F$ has the mixed g-monotone property;

(7) (a) $F$ is continuous or

(b) $X$ has the following properties:

(i) if a nondecreasing sequence $\left\{x_{n}\right\} \rightarrow x$, then $x_{n} \preceq x$ for all $n$,

(ii) if a nonincreasing sequence $\left\{y_{n}\right\} \rightarrow y$, then $y_{n} \geq y$ for all $n$.

Then $g$ and $F$ have a coupled coincidence point. Moreover, if for any $(x, y),(u, v) \in X \times X$ there exists $(w, z) \in X \times X$ such that $(x, y) \preceq_{2}(w, z)$ and $(u, v) \preceq_{2}(w, z)$, then $(s, s)$ is the unique coupled coincidence point of $F$ and $g$.

Note that, in particular if we take $\psi(t)=t$ and $\varphi(t) \equiv 0$ in Theorem 4.7, then the function $\theta$ satisfies the basic assumption of the main theorem of Lakshmikantham and Ćirić [11] and other assumptions are similar. Therefore their main result implies from this theorem. Of course in our theorem we have required the monotonicity of $g$, but this condition ensures the uniqueness of coupled coincidence point.

Now, we present our example. This example illustrates Theorem 4.4. Moreover, the conditions of Theorem 4.4 are fulfilled, but Theorem 2.1 of Alsulami [13] cannot be applied.

Example 4.8 Let $X=[0,2 / 3]$ be endowed with its Euclidian metric $d(x, y)=|x-y|$ and its usual order $\leq$. Take $F: X \times X \rightarrow X$ defined by

$$
F(x, y)=\frac{1}{8}\left\{x^{2}-y^{2}+\frac{3}{2}\right\} \quad \text { for } x, y \in X
$$

and $g: X \rightarrow X$ defined by

$$
g(x)=\frac{x}{2} \quad \text { for } x \in X .
$$

$F$ has the mixed $g$-monotone property. We show that $F$ and $g$ are not compatible. Let $\left\{x_{n}\right\}$ and $\left\{y_{n}\right\}$ be two sequences in $X$ such that

$$
\lim _{n \rightarrow \infty} g\left(x_{n}\right)=\lim _{n \rightarrow \infty} F\left(x_{n}, y_{n}\right)=a \text { and } \lim _{n \rightarrow \infty} g\left(y_{n}\right)=\lim _{n \rightarrow \infty} F\left(y_{n}, x_{n}\right)=b
$$

so $a=b=3 / 16$ and then $\lim _{n \rightarrow \infty} x_{n}=\lim _{n \rightarrow \infty} y_{n}=3 / 8$;

$$
\begin{aligned}
\lim _{n \rightarrow \infty} d\left(F\left(g\left(x_{n}\right), g\left(y_{n}\right)\right), g\left(F\left(x_{n}, y_{n}\right)\right)\right) & =\lim _{n \rightarrow \infty}\left|\frac{1}{8}\left\{\left(\frac{x_{n}}{2}\right)^{2}-\left(\frac{y_{n}}{2}\right)^{2}+\frac{3}{2}\right\}-\frac{x_{n}^{2}-y_{n}^{2}+\frac{3}{2}}{16}\right| \\
& =\frac{3}{32} .
\end{aligned}
$$


Let $\psi(t)=\theta(t)=t$ and $\varphi(t)=t / 3$ for $t \geq 0$. Then

$$
\begin{aligned}
\psi(d(F(x, y), F(u, v)))= & \left|\frac{1}{8}\left\{x^{2}-y^{2}+\frac{3}{2}\right\}-\frac{1}{8}\left\{u^{2}-v^{2}+\frac{3}{2}\right\}\right| \\
= & \frac{1}{8}\left|\left(x^{2}-u^{2}\right)-\left(y^{2}-v^{2}\right)\right| \\
= & \frac{1}{8}|(x-u)(x+u)-(y-v)(y+v)| \\
\leq & \frac{1}{3}\left(\left|\frac{x}{2}-\frac{u}{2}\right|+\left|\frac{y}{2}-\frac{v}{2}\right|\right) \\
= & \frac{1}{3}(d(g(x), g(u))+d(g(y), g(v))) \\
\leq & \frac{2}{3} \max \{d(g(x), g(u)), d(g(y), g(v))\} \\
= & \max \{d(g(x), g(u)), d(g(y), g(v))\} \\
& -\frac{1}{3} \max \{d(g(x), g(u)), d(g(y), g(v))\} \\
= & \theta(\max \{d(g(x), g(u)), d(g(y), g(v))\}) \\
& -\varphi(\max \{d(g(x), g(u)), d(g(y), g(v))\}) .
\end{aligned}
$$

Hence, all the assumption of Theorem 4.4 are satisfied. So we see that $F$ and $g$ have the unique coupled coincidence point $(3 / 8,3 / 8)$.

\section{Competing interests}

The authors declare that they have no competing interests.

\section{Authors' contributions}

All authors contributed equally to the writing of this paper. All authors read and approved the final manuscript.

\section{Author details}

'School of Mathematical Sciences, Faculty of Science and Technology, Universiti Kebangsaan Malaysia, UKM, Bangi,

Selangor Darul Ehsan 43600, Malaysia. ${ }^{2}$ Department of Mathematics, King Abdulaziz University, P.O. Box 138381, Jeddah, 21323, Saudi Arabia.

\section{Acknowledgements}

The authors would like to acknowledge the financial support received from Universiti Kebangsaan Malaysia under the research grants UKM-DIP-2012-31, AP-2013-009 and 'Modal Insan Berpusat (NIC1)'.

\section{Received: 2 June 2014 Accepted: 3 September 2014 Published: 03 Oct 2014}

\section{References}

1. Alsulami, SM, Alotaibi, A: Coupled coincidence point theorems for compatible in partially ordered metric spaces. Bull. Math. Anal. Appl. 4, 129-138 (2012)

2. Alsulami, SM, Hussain, N, Alotaibi, A: Coupled fixed and coincidence points for monotone operators in partial metric spaces. Fixed Point Theory Appl. 2012, 173 (2012). doi:10.1186/1687-1812-2012-173

3. Alsulami, SM, Kozlowski, WM: On the set of common fixed points of semigroups of nonlinear mappings in modular function spaces. Fixed Point Theory Appl. 2014, 4 (2014). doi:10.1186/1687-1812-2014-4

4. Ćirić, L, Alsulami, SM, Parvaneh, V, Roshan, JR: Some fixed point results in ordered $G_{p}$-metric spaces. Fixed Point Theory Appl. 2013, 317 (2013). doi:10.1186/1687-1812-2013-317

5. Hussain, N, Nashine, HK, Kadelburg, Z, Alsulami, SM: Weakly isotone increasing mappings and endpoints in partially ordered metric spaces. J. Inequal. Appl. 2012, 232 (2012). doi:10.1186/1029-242X-2012-232

6. Shaddad, F, Salmi, M, Alsulami, SM: Fixed point theorems in cone metric spaces by using sequentially lower semicontinuous for multivalued maps. Bull. Malays. Math. Soc. (accepted, 2013)

7. Shaddad, F, Salmi, M, Alsulami, SM: Common fixed point results of Ciric-Suzuki-type inequality for multivalued maps in compact metric spaces. J. Inequal. Appl. 2014, 7 (2014). doi:10.1186/1029-242X-2014-7

8. Wang, S, Alsulami, SM, Ćirić, L: Common fixed point theorems for nonlinear contractive mappings in fuzzy metric spaces. Fixed Point Theory Appl. 2013, 191 (2013). doi:10.1186/1687-1812-2013-191 
9. Ran, ACM, Reurings, MCB: A fixed point theorem in partially ordered sets and some applications to matrix equations. Proc. Am. Math. Soc. 132, 1435-1443 (2004)

10. Bhaskar, TG, Lakshmikantham, V: Fixed point theorems in partially ordered metric spaces and applications. Nonlinear Anal. 65, 1379-1393 (2006)

11. Lakshmikantham, V, Ćirić, L: Coupled fixed point theorems for nonlinear contractions in partially ordered metric spaces. Nonlinear Anal. 70, 4341-4349 (2009)

12. Choudhury, BS, Kundu, A: A coupled coincidence point result in partially ordered metric spaces for compatible mappings. Nonlinear Anal. 73, 2524-2531 (2010)

13. Alsulami, SM: Some coupled coincidence point theorems for a mixed monotone operator in a complete metric space endowed with a partial order by using altering distance functions. Fixed Point Theory Appl. 2013, 194 (2013)

14. Harjani, J, Sadarangani, K: Generalized contractions in partially ordered metric spaces and applications to ordinary differential equations. Nonlinear Anal. 72, 1188-1197 (2010)

15. Nieto, JJ, Rodríguez-López, R: Contractive mapping theorems in partially ordered sets and applications to ordinary differential equations. Order 22, 223-239 (2005)

16. Harjani, J, López, B, Sadarangani, K: Fixed point theorems for mixed monotone operators and applications to integral equations. Nonlinear Anal. 74, 1749-1760 (2011)

17. Luong, NV, Thuan, NX: Coupled fixed points in partially ordered metric spaces and application. Nonlinear Anal. 74 983-992 (2011)

18. Alotaibi, A, Alsulami, SM: Coupled coincidence points for monotone operators in partially ordered metric spaces. Fixed Point Theory Appl. 2011, 44 (2011)

19. Razani, A, Parvaneh, $\mathrm{V}$ : Coupled coincidence point results for $(\psi, \alpha, \beta)$-weak contractions in partially ordered metric spaces. J. Appl. Math. 2012, Article ID 496103 (2012)

20. Choudhury, BS, Kundu, A: $(\psi, \alpha, \beta)$-Weak contractions in partially ordered metric spaces. Appl. Math. Lett. 25, 6-10 (2012)

21. Samet, B, Karapınar, E, Aydi, H, Ćojbašić Rajić, V: Discussion on some coupled fixed point theorems. Fixed Point Theory Appl. 2013, 50 (2013)

10.1186/1687-1812-2014-204

Cite this article as: Shaddad et al.: Coupled point results in partially ordered metric spaces without compatibility. Fixed Point Theory and Applications 2014, 2014:204

\section{Submit your manuscript to a SpringerOpen ${ }^{\circ}$ journal and benefit from:}

- Convenient online submission

- Rigorous peer review

- Immediate publication on acceptance

- Open access: articles freely available online

- High visibility within the field

- Retaining the copyright to your article 